ESAIM: PROCEEDINGS AND SURVEYS, November 2014, Vol. 46, p. 83-85

ECIT 2012 - Witold Jarczyk, Daniele Fournier-Prunaret, João Manuel Gonçalves Cabral

\title{
ON THE HISTORY OF ONE-DIMENSIONAL DYNAMICS
}

\author{
A.N.SHARKOVSKY ${ }^{1}$
}

\begin{abstract}
We discuss the development of one-dimensional dynamical systems theory, in particular, chaotic dynamics in the "pre-chaos epoch". Mostly it will be about author's results on attractors of trajectories and the structure of their basins.

Résumé. Nous discutons le developpement de la theorie des systemes dynamiques a une dimension, en particulier, dynamique chaotique dans le "pre-chaos" epoque. Surtout, il sera sur les resultats de l'auteur sur attracteurs de trajectoires et la structure de leurs bassins.
\end{abstract}

AMS (2010) subject classification: $37-03,37 \mathrm{~B}, 37 \mathrm{E}$

Keywords: history of one-dimensional dynamical systems; chaotic dynamics; cycle; $\omega$-limit set; complexity of dynamical system

Most clefs: histoire des systemes dynamiques a une dimension; dynamique chaotique; cycle; $\omega$-limit ensemble; complexite de systeme dynamique

At present time one-dimensional dynamical systems takes up a significant place not only in the theory of dynamical systems but in contemporary dynamics as well. As is now well known, one-dimensional systems have a wide range of dynamical behavior up to the regimes where the behavior of trajectories (of a deterministic system) are virtually undistinguishable from the behavior of random processes. From the viewpoint of the descriptive theory of sets, these systems are just as complex as dynamical systems on arbitrary spaces! At the same time, due to the simplicity of the phase space, the theory of one-dimensional systems is now highly developed; it contains deep results and effective criteria, which can be accompanied with simple and visual computer experiments.

40-50 years ago the attitude to 1D maps and dynamical systems with discrete time was distinctly different from that which is today. One of the reasons is of course that dynamical systems theory was originated as the qualitative theory of differential equations. But there were also other reasons. For example, Ya.G. Sinai in the book [11] wrote on the page 103: "About twenty years ago I had a general feeling that the structure of one-dimensional dynamical systems is relatively straightforward and can be understood until the end, and at the same time, the results valid for one-dimensional case, have not natural many-dimensional analogues. Subsequent years have shown that both of these feelings are wrong."

One example from my own experience. International conferences on nonlinear oscillations, that held 30-50 years ago, were of very importance in developing both nonlinear dynamics and one-dimensional dynamical systems in particular. I first participated at such conferences in 1967 in Prague where I submit my talk on the coexistence of periodic solutions for the difference equation $x(n+1)=f(x(n))$ [9]. But only the abstract of

${ }^{1}$ Institute of Mathematics,

National Academy of Sciences of Ukraine,

e-mail: asharkov@imath.kiev.ua

(C) EDP Sciences, SMAI 2014 
my report had been included in the proceedings: at that time "simple" difference equations seemed far away from the problems of serious nonlinear oscillations theory.

The second half of the 70s was a turning point for one-dimensional dynamical systems. During this period works had appeared which greatly enriched the subject matter of the theory of dynamical systems, and not just one-dimensional. In particular, new notions such as "chaos", "sensitive dependence on initial conditions" had been introduced, various universal properties of smooth systems had been discovered, for example, that describing the speed of bifurcations (and not just their order, which was known earlier). Simple and beautiful laws linking the old and new notions had been found. My paper [4] had lastly attracted attention, its translation had been published [12]. Presently we can say that at this period one-dimensional dynamical systems played a key role in developing the whole theory of dynamical systems. And now interest in one-dimensional dynamics is steadily growing, and not only among mathematicians.

The notion "chaos" as a mathematical term first appeared in 1975 in the work of American mathematicians T.Li and J.Yorke [2] just in conformity to one-dimensional maps. And how about the development of onedimensional chaotic dynamics before 1975, in the "pre-chaos epoch"?

Now, use has been made of many distinct mathematical definitions of chaos but all these approve the presence of a lot of trajectories with fundamentally different behavior. And first of all, a wide variety of cycles and, in general, $\omega$-limit sets says about a diversity of the asymptotical behavior of trajectories. Just these problems for one-dimensional maps were considered in my works of 1961-1966 years and in my PhD thesis "Some problems of the theory of one-dimensional iteration processes" (1961) and DSc thesis "On $\omega$-limit sets of discrete dynamical systems" $(1966)^{1}$. In particular, the part of the known reordering of all natural numbers, namely, $\quad 1 \prec 2 \prec 2^{2} \prec \ldots \prec 2^{m} \prec 2^{m+1} \prec \ldots \forall \neq 2^{k}$ for any $m, k \geq 0$, was represented already in [3] and the $\mathrm{PhD}$ thesis.

In the DSc thesis the results on the coexistence of cycles occupied very small part and an essential part of this work is devoted to the coexistence of invariant infinite (in particular, Cantor) sets.

As is now well known, if a map $f \in C^{0}(I, I)$ possesses a periodic point of period not equal to a power of two then the dynamical system is complex, there exist cycles of arbitrarily large periods, homoclinic trajectories, chaos in the Li-Yorke sense, and the topological entropy is positive.

In the thesis and, in particular, in [7,10], many other properties characterizing the complexity of a dynamical system are pointed out. These use just the notion of $\omega$-limit set (that is, attractor) of trajectory. It is convenient to distinguish three kind of $\omega$-limit sets, namely: 1) maximal $\omega$-limit sets, which is not contained in a larger $\omega$-limit set, 2) locally maximal ones, for each there exists vicinity not containing a larger $\omega$-limit set, and 3 ) the others (for which any their vicinity contains a larger $\omega$-limit set). If a map $f$ has a cycle of period $\neq 2^{k}, k \geq 0$, then this map has at least one maximal $\omega$-limit set containing cycles. Let $A_{\max }$ be such an $\omega$-limit set (it is a Cantor set or several intervals) and let $\mathfrak{M}$ be the set of all $\omega$-limit sets contained in $A_{\text {max }}$ (i.e. $\mathfrak{M}=\left\{A(x) \subseteq A_{\max }, x \in I\right\}$, where $A(x)$ is for the $\omega$-limit set of trajectory through $x$ ).

The set $\mathfrak{M}$ is of continuum power, moreover, even its subset consisting of minimal $\omega$-limit sets different from cycles (thus, each is a Cantor set) has continuum power. Every chain $\mathfrak{L}: \ldots \supset A^{\prime} \supset A^{\prime \prime} \supset \ldots, A^{\prime}, A^{\prime \prime} \in \mathfrak{M}$, being maximal (i.e., not being a part of a longer such chain) is countable or has continuum power. The set $\mathfrak{M}^{\prime} \subset \mathfrak{M}$ which consists of all locally maximal $\omega$-limit sets contained in $A_{\max }$, has continuum power but contains only a countable number of minimal $\omega$-limit sets (each of which is a cycle). Every $A \in \mathfrak{M}^{\prime}$ is a Cantor set or a cycle. Every maximal in $\mathfrak{M}^{\prime}$ chain $\mathfrak{L}^{\prime}: \ldots \supset A^{\prime} \supset A^{\prime \prime} \supset \ldots, A^{\prime}, A^{\prime \prime} \in \mathfrak{M}^{\prime}$, is countable, and moreover, is similar to the set of rational numbers: for any $A^{\prime}$ and $A^{\prime \prime} \in \mathfrak{L}^{\prime}$ there exists $A^{\prime \prime \prime} \in \mathfrak{L}^{\prime}$ laying between $A^{\prime}$ and $A^{\prime \prime}$ (if $A^{\prime} \supset A^{\prime \prime}$ then $A^{\prime} \supset A^{\prime \prime \prime} \supset A^{\prime \prime}$ ). If $B(A)=\{x \mid A(x)=A\}$ is the basin of attraction of $A$ and $A \in \mathfrak{M} \backslash \mathfrak{M}^{\prime}$ then $B(A)$, being $F_{\sigma \delta^{-}}$set but not $G_{\delta \sigma^{-}}$-set, is a third Baire class set.

One often hears the argument that in the 60-70s one or other noteworthy result (in particular, concerning the cycles coexistence) was unavailable or not widely known in the West because of this result was published

\footnotetext{
${ }^{1}$ Recently the full text of this DSc thesis is published as a part of my book "Attractors of trajectories and their basins", Naukova Dumka, Kiev, 2013, 320pp. (in Russian).
} 
in Russian and in a not easily accessible journal. As to my results on one-dimensional maps, on the complex dynamics these generate, almost all of them were published in English as early as the 60s (see [3,5-8]).

In conclusion, a few words about my first meetings with J.Yorke, happened and not happened. Our personal contact occurred in 1975 at the seventh International conference on nonlinear oscillations (ICNO-1975) in Berlin. This meeting is mentioned, not even in one publication, but it is presented quite original. For example, in [1] one can read: "... there is an amusing anecdote linking these works ["Coexistence of cycles ..." [4] and "Period three implies chaos" [2]]. Sharkovsky had published his theorem in a Ukrainian journal (in Russian) in the early sixties, but at the time nobody had taken notice of it. According to Gleick [Chaos. Making a New Science, Penguin, 1987], Sharkovsky approached Yorke at a conference in East Berlin. There was something very important the Ukrainian had to tell Yorke. In true cold war fashion, a meeting was arranged on a boat on the Spree River. There, with the help of an anonymous Polish translator, Sharkovsky informed Yorke about his discovery ..."

In reality, our meeting took place on a ship during a joint walk of conference participants on the Spree River. Seeing Yorke is not far away, I wanted to explain him that results which are stronger than those represented in his report were obtained for more than 10 years ago. On a piece of paper I wrote $3,5,7, \ldots, 4,2,1$, and realizing that my very poor English will be useless, I asked for help with translation my familiar Pole Marian Kwapisz, but he knew at the time only French, so I had to invite also Frenchman Christian Mira, who spoke a little English. Of course, such a binary translation hardly could be successful.

Our personal meeting with Yorke, however, could have take happened before, early as 1969, during the fifth International conference on nonlinear oscillations (ICNO-1969) in Kiev. This conference numbered about 500 participants, among which were many mathematicians from the U.S.A. J.Yorke also sent the abstract of his report, and the report was included in the section "Qualitative methods" (where I was not only as a speaker, but as a scientific secretary). However, Yorke had not arrived to Kiev, but later he sent the text of his report, which was published in the proceedings of the conference [13].

\section{REFERENCES}

[1] Alejo Barrio Blaya and Victor Jimenez Lopez, Is trivial dynamics that trivial?, Amer. Math. Monthly 113, 2006, $109-133$.

[2] T.-Y. Li \& J.A.Yorke, Period three implies chaos, Amer. Math. Monthly 82, 1975, 985-992.

[3] A.N.Sharkovsky, The reducibility of a continuous function of a real variable and the structure of the fixed points of the corresponding iteration process, Dokl. Akad. Nauk USSR, 139 (1961), no.5; Soviet Math. Dokl., vol. 2 (1961).

[4] A.N.Sharkovsky, Coexistence of cycles of a continuous map of the line into itself, Ukrain. Mat. Zh. 16, no.1 (1964), 61-71 (in Russian); Intern. J. Bifurcation and Chaos 5, 5, 1995, 1263-1273; Proceed. conf. "Thirty years after Sharkovskii's theorem: new perspectives", World Scientific, Ser. Nonlinear Sci., Ser. B, 8, 995, 1-11.

[5] A.N.Sharkovsky, On attracting and attracted sets, Dokl. Akad. Nauk USSR, 160 (1965), No.5; Soviet Math. Dokl. vol. 6 (1965).

[6] A.N.Sharkovsky, A Classification of fixed points, Ukrain. Mat. Zh. 17, no.5 (1965); Amer. Math. Soc. Transl. 97, no.2, 1970, 159-179.

[7] A.N.Sharkovsky, The partially ordered system of attracting sets, Dokl. Akad. Nauk USSR, 170 (1966), no.6; Soviet Math. Dokl. vol. 7 (1966), no.5.

[8] A.N.Sharkovsky, Behavior of a mapping in the neighborhood of an atrracting set, Ukrain. Mat. Zh. 18, no.2 (1966); Amer. Math. Soc. Transl. 97, no.2, 1970, 227-258.

[9] A.N.Sharkovsky, On the periodic solutions of a class of finite differences equations, Proceed. Fourth conference on nonlinear oscillations, Academia, Prague, 1968, p. 249.

[10] A.N.Sharkovsky, On the problem of isomorphism of dynamical systems, Proc. V Intern. conf. on nonlin. oscillations, Naukova dumka, Kiev, vol. 2, 1970, 541-545 (in Russian).

[11] Ya.G. Sinai, Modern problems of ergodic theory, Fizmatlit, Contemporary Mathematics, vol. 31, 1995.

[12] P.Stefan, A theorem of Sharkovskii on the existence of periodic orbits of continuous endomorphisms of the real line, Commun. Math. Phys. 54, 1977, 237-248.

[13] J.A.Yorke, Non-Lipshitz Lyapunov functions, Proc. V Intern. conf. on nonlin. oscillations, Naukova dumka, Kiev, vol. 2, 1970, $170-176$. 